\title{
Developing an efficient clinical information system for a comprehensive psychiatric institute: I. Principles, design, and organization
}

\author{
JUAN E. MEZZICH, JOHN T. DOW, and GERALD A. COFFMAN \\ Western Psychiatric Institute and Clinic, University of Pittsburgh School of Medicine \\ Pittsburgh, Pennsylvania 15261
}

\begin{abstract}
The principles, design considerations, and components of a clinical information system intended to support and enhance the patient care, educational, and research missions of a comprehensive psychiatric institute are presented. Major characteristics of the system are its emphases on efficiency, simplicity, and intensive user involvement throughout the development of the system.
\end{abstract}

The purpose of this paper is to describe the principles and development of an efficient and computerized clinical information system (CIS) for a comprehensive psychiatric institute. This system, in its final form, will support the various missions of the Western Psychiatric Institute and Clinic (WPIC). These activities include psychiatric care for child, adolescent, adult, and geriatric patients through several specialized treatment modules involving 1,600 inpatients and 124,000 outpatient visits per year, as well as the related educational and research works of the Department of Psychiatry of the University of Pittsburgh School of Medicine.

\section{BACKGROUND}

The early expectations for mental health information systems might be characterized by Rome's (1967, p. 451) hopeful statement: "Psychiatry is now on the threshold of a fourth quantum advance. Automation of informationprocessing will achieve what never has been available heretofore-a valid data-base for psychiatry's assumptions, treatments, logistics, and at the same time it offers a potential solution for psychiatry's administrative complexities."

Since Rome's (1967) lofty expectations for the use of computerized CISs in psychiatry, there have been a number of papers containing encouraging reports on actual developmental efforts (e.g., Gianturco \& Ramm, 1971; Sletten, 1974; Strotz, Malerstein, \& Starkweather, 1969). More recently, critical reviews of experiences in design and development have been published (Hedlund, Sletten, Evenson, Altman, \& Won Cho, 1977; Klein,

A preliminary version of this paper was presented at the annual meeting of the Professional Association for the Development, Use, and Management of Information Systems in Higher Education (CAUSE), Orlando, Florida, November 27-30, 1979.
Greist, \& VanCura, 1975; Leader \& Klein, 1977). These papers point out that CIS design has reached neither Rome's high hopes nor the more practical expectations of early developmental efforts.

The reasons for not achieving these early expectations can be traced to a number of factors. Probably the most important of these is that many CISs are not well accepted by their intended clinician users. One reason for poor acceptance is the use of forms that are almost fully structured, allowing no or little space for narrative and flexible description of the patient's condition. Other CISs use two separate forms: one completely standardized and the other containing a narrative description of the patient (Laska, 1974). These require a significant amount of duplicate clinical reporting, which may result in incomplete documentation on at least one of the forms.

Other factors for lack of acceptance have included, on one hand, difficulties experienced by clinical evaluators conducting rigidly structured interviews or recording clinical data on grossly unfamiliar formats and, on the other hand, complaints by the treatment staff and other users about irrelevance of the informational content, difficulty of appraising a voluminous package of forms, untimely retrieval of needed information, and concerns about both privacy and confidentiality and the presumed dehumanizing aspects of computerization (Hedlund, Evenson, Sletten, \& Won Cho, 1980; Hedlund et al., 1977).

Finally, one of the major factors underlying poor user acceptance appears to be that CISs have been typically set or imposed "from the top down," with no or little involvement of the clinical staff, especially front-line clinicians, in systems development.

The above-mentioned problems have contributed to prevent many CISs' living up to their potential. Consequently, new developmental efforts must attempt to 
work out and use improved methodology for systems design, implementation, and monitoring.

\section{OVERVIEW OF THE WPIC CLINICAL INFORMATION SYSTEM}

\section{Origins, Auspices, and Aims}

The WPIC approach has as one of its antecedents the considerable amount of systems thinking and experience that have taken place at this psychiatric institute during the past several years (Dow, 1975; Henderson, Magnussen, Snyderman, \& Homann, 1975; Kupfer, Levine, \& Nelson, 1976). Other antecedents include the KDS system (Kupfer \& Detre, 1971) and the Comprehensive Psychiatry Assessment Schedule (Mezzich, Note 1). These batteries are mostly structured and are addressed to an integral evaluation of general psychiatric patients. Another important antecedent is the patient data system. This is a computerized procedure for uniform patient registration and for the systematic collection and retrieval of demographic and financial information. The data are collected by financial interviewers and are entered on-line at CRTs during the admitting interview. It was developed in 1975, and since then it has been fully operational and undergoing periodic revisions and improvements in collaboration with an administrative users' group. Another relevant antecedent is the discharge abstracting procedure (PCAP) developed by Huber, Darragh, and colleagues. Finally, WPIC has had a unit record system for medical records since 1935.

In September 1978, a committee consisting of representatives from the major clinical, research, and administrative programs at WPIC was formed to explore the development of a system for improved clinical evaluation, monitoring, and communication throughout the institute. After some preliminary deliberations, the committee decided to focus attention first on the initial evaluation process as a reasonable and manageable starting point for building a patient-centered CIS. A working group composed of four clinical and epidemiological psychiatrists and an information scientist was then formed to design an initial evaluation procedure. After basic developmental efforts, another systems analyst and a systems specialist were recruited to enhance the development of the initial evaluation procedure and the overall design and implementation of the CIS.

\section{Design Considerations}

Five design considerations were regarded as important for the successful development of a CIS. These include user involvement, simplicity, reliability, validity, and confidentiality. In the following subsections, factors that can influence each of these and the approach taken for their implementation are summarized.

User involvement. It was felt that the best way to achieve clinician acceptance, perhaps the most crucial issue for a system's success, was to attempt to involve the potential users in the design of the system as extensively and intensively as possible. This was implemented first through a series of CIS concept meetings with members of 38 different clinical care, research, and support programs at WPIC, including staff from both line and supervisory levels. The results from these discussions were then systematized by asking these individuals and groups to complete a questionnaire designed to provide a comprehensive view of user requirements, with special emphasis on the initial evaluation. Users also participated in the selection of items for CIS forms, and the related pilot testing, in-service training, and monitoring.

To ensure the continued support of key clinical leadership, a conscientious effort has been made to maintain good communication with them, through formal and informal meetings. This was thought necessary to identify changing requirements for future revisions of the system.

Simplicity. A clinical information procedure should be simple, brief, and perceived as convenient by clinical evaluators. It is difficult to overemphasize this point, given both the typically busy pace of most clinical settings and the resistance to bulky and overstructured instruments shown by most clinicians. In fact, one report (Leader \& Klein, 1977) indicates that this was a decisive factor in the failure of a computerized system.

Reliability of data. Reliability refers here to the extent with which the patient evaluation process yields accurate results. Sources of variability include the patient, assessment occasion, information gathering procedure, diagnostic system, the evaluators' background and orientation, and data-entry procedure. Each of these is described below.

Patient and occasion. Patient variability refers to changes in the patient's condition over time and his consistency and accuracy as an informant. Occasion variability refers to the environmental setting and time of the evaluation. Both patient and occasion variability are difficult to control by the clinical evaluators. Approaches to solving problems in these areas may include expanding the sources of information to include relatives, associates, and previous clinical records and a routine process of longitudinally evaluating patients during care.

Information gathering procedure. This refers to variability in the ways the patient and other informants are interviewed, the particular set of informational items covered, and the criteria used for determining the presence and intensity of clinical features.

Diagnostic system. This refers to the procedure for using the clinical information gathered to arrive to an assessment summary or diagnostic formulation.

Evaluators' background and orientation. Different theoretical approaches to psychopathology can result in disagreement between evaluators. This source of disagreement may be reduced by setting an evaluation and 
diagnostic framework that is as descriptive and uninferential as possible, and then training the evaluators in its use.

Data-entry procedure. Undesirable variability will result if the data are not consistently recorded or errors are made in coding and entering data in to the system. This source of unreliability may be reduced by having a systems specialist, trained in psychopathological description, check the consistency and completeness of each evaluation form before entering the data into the system. If there are any problems, the clinician that performed the evaluation should be asked to complete pending ratings and make appropriate adjustments.

Validity. Validity refers to the extent to which the CIS fulfills the purposes for which it was designed (e.g., its ability to convey a useful description of the patient's condition and to monitor his care and course). There are two main issues that must be considered to enhance the validity of a CIS.

The first involves the adequacy of the data for clinical description and monitoring. This affects treatment planning, prediction of outcome, and research applications (Klein et al., 1975). Steps that may be taken to enhance the usefulness of the data include the following: (1) Tracking the changing needs of the clinical programs in order to continually update the system and (2) using improved procedures for classification of psychiatric patients, such as the DSM-III (American Psychiatric Association, 1980) and multiaxial diagnostic systems (Mezzich, 1979, 1980), in order to provide a more effective summary description of the patient's condition.

The second issue concerns the acceptability of the system by the clinicians using it, which provides a basic face validity to the system. This point was briefly reviewed earlier regarding the need for user involvement.

Confidentiality. With the increased use of computers in psychiatry, as in other fields, there has been a growing public awareness and concern about the level of confidentiality that computerized systems can maintain. These concerns about psychiatric data are based on the traditional element of trust established between the clinician and the patient. One aspect of trust includes the patient's belief that all data collected will be for beneficial purposes. Many times a clinician consults others about a case, which results in a transfer of data from the patient. In this situation, the clinician is acting in the best interest of the patient. Indirectly beneficial data transfers are involved in research and administrative summary reports. In these cases, each individual patient's identity can be concealed. Harmful data transfers can result when information is accessed by individuals not primarily interested in the best interests of the patient. An example is the situation in which a past history of psychiatric illness is unwarrantedly used to deny employment (Baldwin, Leff, \& Wing, 1976). A more flagrant example is the use of clinical information for defamatory purposes.

There are reports about clinicians refusing to cooper- ate with a computerized CIS because they felt that confidentiality was not maintained (Curran, Laska, Kaplan, \& Bank, 1973). On the other hand, if confidentiality is maintained too tightly, information useful to the patient, to the advancement of psychiatric knowledge, and to the administration of the mental health system may not be available. Clearly, a balance must be struck between the level of confidentiality and the requirements for clinical work.

The steps taken to provide security for the CIS at WPIC include the use of an in-house computer system. The data in this system are protected through the use of assigned and frequently changed passwords. Confidentiality is further promoted by restricting the access of a user to the type of data he needs and is authorized to have and by implementing adequate physical security. A similar procedure has been used on other systems and seems to provide the proper balance between confidentiality and beneficial use of the data (Curran et al., 1973).

\section{Overall Architecture}

The design of the WPIC CIS reflects the patient care organization of the psychiatric institute. Figure 1 shows the various patient flow and patient care phases at the institute and the CIS form intended to organize and document each phase. The organization of treatment programs into specialized modules (affective disorders, schizophrenia, special therapies, adolescent and young adult, and geriatric psychiatry), each consisting of an inpatient and an outpatient component, was recently implemented at WPIC. Two child inpatient units within the institute and a large child outpatient program at the adjacent and affiliated Pittsburgh Child Guidance Center, which are also admission and referral options for patients applying for care at the WPIC entry station, the Diagnostic and Evaluation Center, are not shown in Figure 1. The conceptualization of the key CIS forms identified in Figure 1 reflects the judgment of the CIS organizing group and recommendations from the WPIC medical records committee and external agencies such as the Joint Commission on Accreditation of Hospitals (1979).

The first component of the WPIC clinical information system is the initial evaluation form (IEF). It organizes and documents the initial and basic assessment of each patient applying for care. Its basic structural feature is the complementary interspersing of narrative and standardized components. It is currently in its final stage of development and is described later in a companion paper to illustrate the WPIC approach to system design and implementation.

The set of modular evaluation forms constitute second-step evaluation procedures specific for each one of the treatment modules to which patients can be currently assigned at WPIC: affective disorders, schizophrenia, special therapies, geriatric psychiatry, adolescent and young adult. and, through special channels, child psychiatry. Each specific modular evaluation form will 


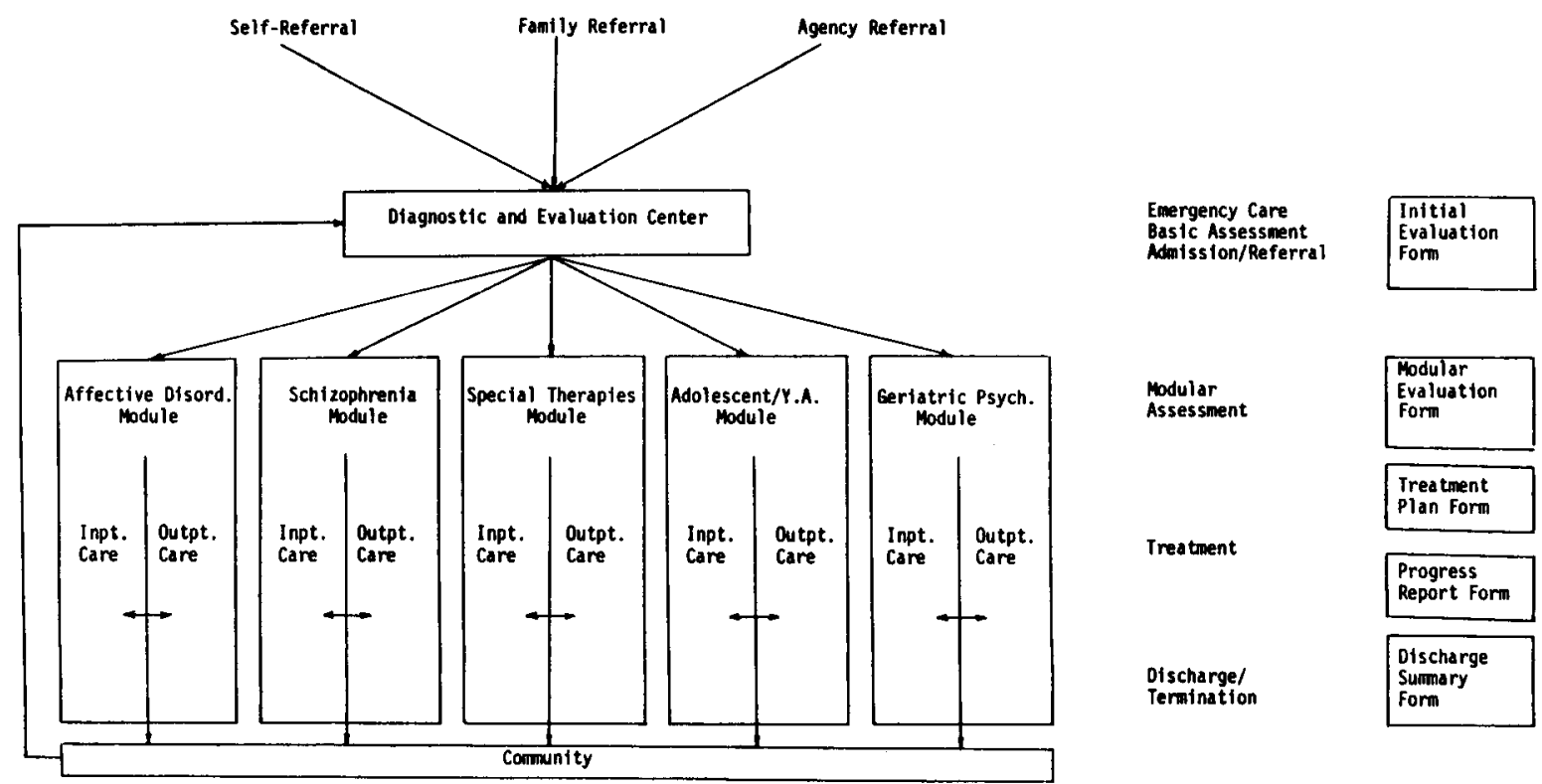

Figure 1. Patient care diagram and clinical information system forms at the Western Psychiatric Institu te and Clinic.

expand on the IEF data base to include detailed information relevant to the particular clinical features of the patient population in the module and to the treatment strategies emphasized there.

A treatment plan form and a progress report form of semistandardized format will be developed to document the main treatment phase of patient care. The treatment plan form will use as a starting point the data bases provided by the IEF and the appropriate module evaluation form, and it will deal systematically with the articulation of codified patient problems with set treatment goals, performance criteria, treatment methods, and goal attainment dates. It will systematize further and build on a noncodified treatment plan form recently developed and implemented at WPIC. The progress report form will log the course of the patient under treatment along the lines set in the treatment plan form. For its development, we will use the experience obtained with the current contact sheet, which includes standardized billing information and space for a narrative progress note. Progress report forms have traditionally been so idiosyncratic in content and entrenchedly narrative in form that partial standardization of the progress report form will constitute a major challenge in the development of the CIS.

The discharge summary form will encompass the discharge or termination summary prepared at the end of an inpatient or outpatient treatment episode. This form will be structurally and longitudinally integrated with the CIS forms corresponding to the earlier phases of clinical care.

In addition to the above-mentioned forms docu- menting key aspects of clinical evaluation and treal ment, a patient tracking system is being developed $t_{1}$ monitor, for each patient, dates and settings of majo evaluations, referral and actual admission to eithe inpatient or outpatient care, interunit transfers, anı discharge or termination of a treatment episode.

\section{COMMENT}

A question that may be asked is, "How is this syster really different from other mental health informatio systems developed at similar institutions?" One majc characteristic of the CIS being developed at WPIC its emphasis on efficiency. This is reflected in th parsimony of the content of the evaluation forms and $i$ the mutually complementary arrangement of the narr: tive and standardized components of the evaluatio format to facilitate its completion. Another majc differential feature of the WPIC system is intensive use involvement throughout the design, implementatior and monitoring phases of the system.

Extensive trial runs have already documented th acceptability and usefulness of the Initial Evaluatio: Form. Furthermore, the emphases on efficiency and use involvement appear to increase the likelihood that th clinical information system will live up to its potentia and expectations. A challenge remains, however, fo both the individuals responsible for the system and th institution to make appropriate adjustments on the com ponents of the system already implemented and t complete its overall design. 


\section{REFERENCE NOTE}

1. Mezzich, J. E. Manual of the comprehensive psychiatric assessment schedule (technical report). Stanford, Calif: Stanford University, Department of Psychiatry and Behavioral Sciences, 1977.

\section{REFERENCES}

American Psychiatric Association. Diagnostic and statistical manual of mental disorders (3rd ed.) (DSM-III). Washington, D.C: Author, 1980.

Baldwin, J. A., LefF, J., \& Wing, J. K. Confidentiality of psychiatric data in medical information systems. British Journal of Psychiatry, 1976, 128, 417-427.

Curran, W. J., Laska, E. M., Kaplan, H., \& Bank, R. Protection of privacy and confidentiality. Science, 1973, 182, 797-801.

Dow, J. T. Designing computer software for information systems in psychiatry. Computers in Biomedical Research, 1975, 8, 538-559.

Gianturco, D. T., \& RAMm, D. A conversational psychiatric information network. Community Mental Health Journal, 1971, 2, 127-133.

Hedlund, J. L., Evenson, R. C., Sletten, I. W., \& Won Cho, D. The computer and clinical prediction. In J. B. Sidowski, J. H. Johnson, \& T. A. Williams (Eds.), Technology in mental health care delivery systems. Norwood, N.J: Abley, 1980.

Hedlund, J. L., Sletten, I. W., Evengon, R. C., Altman, H., \& Won Chо, D. Automated psychiatric information systems: A critical review of Missouri's standard system of psychiatry. Journal of Operational Psychiatry, 1977, 8, 5-26.

Henderson, P. B. Magnussen, M. G., Snyderman, B. B., \& Homann, J. A clinical decision-making model for child psy- chiatric intervention selection. Psychological Reports, 1975, 37, 923-934.

Joint Commission on Accreditation of Hospitals. Consolidated standards for child, adolescent, and adult psychiatric, alcoholism, and drug abuse programs. Chicago: Author, 1979.

Kle in, M. H., Greist, J. H., \& VanCura, L. J. Computers and psychiatry: Promises to keep. Archives of General Psychiatry, $1975,32,837-843$

Kupfer, D. J., \& Detre, T. P. KDS systems. New Haven, Conn: Kupfer-Detre Systems, 1971.

Kupfer, D. J., Levine, M. S., \& Nelson, J. A. Mental health information systems: Design and implementation. New York, N.Y: Marcel Dekker, 1976.

LASKA, M. L. The multi-state information system. In J. L. Crawford, D. W. Morgan, \& D. T. Gianturco (Eds.), Progress in mental health information systems: Computer applications. Cambridge, Mass: Ballinger, 1974.

LEADER, M. A., \& KLEIN, D. F. Reflections on instituting a computerized psychosocial history in a clinical facility. Comprehensive Psychiatry, 1977, 18, 489-496.

Mezzich, J. E. Patterns and issues in multiaxial psychiatric diagnosis. Psychological Medicine, 1979, 9, 125-137.

Mezzich, J. E. Multiaxial diagnostic systems in psychiatry. In H. I. Kaplan, A. M. Freedman, \& B. J. Sadock (Eds.), Comprehensive textbook of psychiatry (3rd ed.). Baltimore: Williams \& Wilkins, 1980.

Rome, H. P. Prospects for a psi-net: The fourth quantum advance in psychiatry. Comprehensive Psychiatry, 1967, 8, 450-454.

Sletten, S. W. Using an automated data system for planning and delivery of services. Hospital and Community Psychiatry, 1974, 25, 83-85.

Strotz, C. R., Malerstein, A. J., \& Starkweather, J. A. Automated psychiatric patient record system. Archives of General Psychiatry, 1969, 21, 311-319. 